\title{
Synthesis of Acyclic Insect Pheromones from Cycloalkanones via
}

\section{Acetylenic Lactones}

\author{
Jaswant Rai Mahajan,* Inês Sabioni Resck \\ Departamento de Química, Universidade de Brasilia, 70.910-900 Brasilia - DF, Brazil
}

Received: August 28, 1996

\begin{abstract}
A Fig. 1 descreve um método geral para preparação de feromônios de insetos.
Vários componentes de feromônios de insetos, junto de seus isômeros geométricos e/ou posicionais, foram preparados a partir de Z-lactonas 2a-d, que são facilmente acessíveis pela hidrogenação estereosseletiva (Lindlar Pd, $\mathrm{H}_{2}$ ) dos precursores acetilênicos 1a-d, sendo estes já sintetizados a partir de cicloalcanonas. A isomerização de acetatos de Z-alquenilas 6a-d aos respectivos esteres de E-alquenilas 8a-d, foi realizada tanto pela técnica catalítica $\left(\mathrm{NaNO}_{2}\right.$, $\mathrm{HNO}_{3}, \Delta$ ) como pelo método de inversão química (NBS, TFA; NaI, DMF, $\Delta$ ). O álcool (E)-6-decenílico (7b) foi também preparado diretamente do ester acetilênico (15b) pela redução, em trans, com hidreto de lítio-alumínio $\left(\mathrm{LiAlH}_{4}\right)$, por refluxo em diglima. Os acetatos de decila (18) e hexadecila (19), componentes dos feromônios de mariposa do nabo (Agrotis segetum; turnip moth) e da borboleta Lycorea ceres ceres, respectivamente, foram obtidos pela hidrogenação catalítica $\left(\mathrm{Pd}, \mathrm{H}_{2}\right)$ dos acetatos de Z-alquenilas correspondentes.
\end{abstract}

Figure 1 describes a general method for the preparation of insect pheromones.

Several members of the title compounds, along with their geometric and/or positional isomers, have been prepared from Z-lactones 2a-d, which are easily available from the corresponding acetylenic lactones 1a-d, prepared earlier from cycloalkanones. The $\mathrm{Z}$ to $\mathrm{E}$ isomerization of alkenyl acetates 6a-d to 8a-d was carried out both by a catalytic technique $\left(\mathrm{NaNO}_{2}, \mathrm{HNO}_{3}\right.$, $\Delta$ ) and the chemical inversion procedure (NBS, TFA; NaI, DMF, $\Delta$ ). (E)-6-Decenyl alcohol (7b) was also prepared from the acetylenic ester 15b by the trans reduction with $\mathrm{LiAlH}_{4}$ in refluxing diglyme. decyl acetate (18) and hexadecyl acetate (19), pheromone components of the turnip moth (Agrotis segetum) and the male butterfly Lycorea ceres ceres, respectively, were obtained by the catalytic hydrogenation $\left(\mathrm{Pd}, \mathrm{H}_{2}\right)$ of the corresponding Z-alkenyl acetates.

Keywords: insect pheromones, Z-lactones, acetylenic lactones, cycloalkanones

\section{Introduction}

Pheromones are an important and a rapidly growing class of biologically active organic compounds. A large proportion of pheromones isolated from a variety of insects exemplifies diversely functionallized acyclic compounds, which have been synthesized by several routes ${ }^{1-3}$. While earlier methodologies employed for the construction of the acyclic skeleton involved mainly the acetylenic chemistry and olefin formation by the Wittig or related reactions ${ }^{1}$. More recently, there has been an increasing emphasis on the use of diverse methods for improved isomeric purity of the $\mathrm{E}$ or $\mathrm{Z}$ product and for those leading to the optically active pheromones ${ }^{2,3}$.
We have recently initiated a different approach to these acyclic compounds by the systematic elaboration of cycloalkanones, where the variable size of the starting ketone is exploited to generate the triple or the double bond of the desired product in a regiospecific and stereoselective manner $^{4}$ (Scheme 1).

We have already reported our preliminary results on the conversion of cyclohexanone, involving the transformation of an $\alpha$-chloroketone function into an acetylenic linkage ( $\mathrm{RCHClCOR} \rightarrow \mathrm{RC} \equiv \mathrm{CR}$ ), to both the $\mathrm{Z}$ and $\mathrm{E}$ series of $\mathrm{C}_{9}$ pheromones ${ }^{5}$. A recent publication ${ }^{6}$ described the preparation of $\mathrm{Z}$ and $\mathrm{E}$ macrolides from the acetylenic lactones ${ }^{7}$ 1a-d, whilst we report their transformation into several 


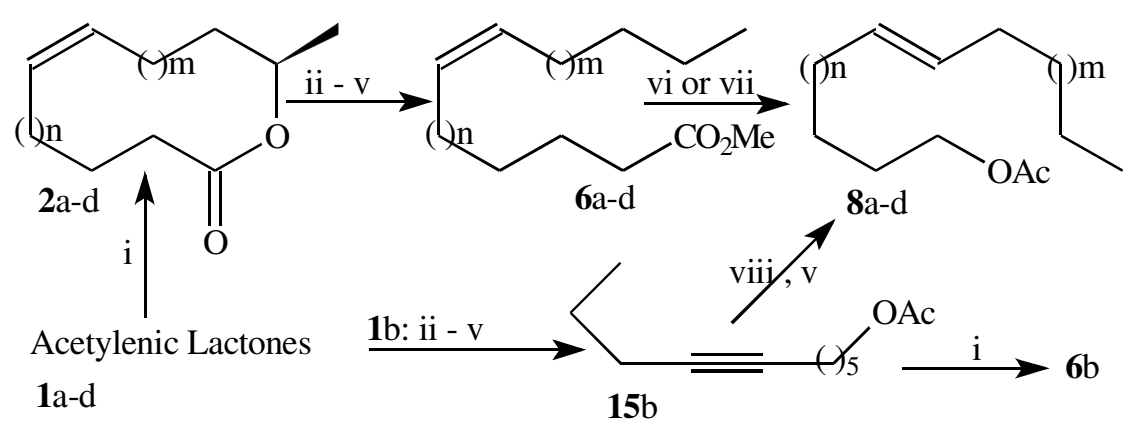

a: $m=n=1 ; b-d: m=0 ; b: n=2 ; c: ~ n=4 ; d: n=8$. i-viii: $90-95 \%$ crude yields, see text.

Figure 1.
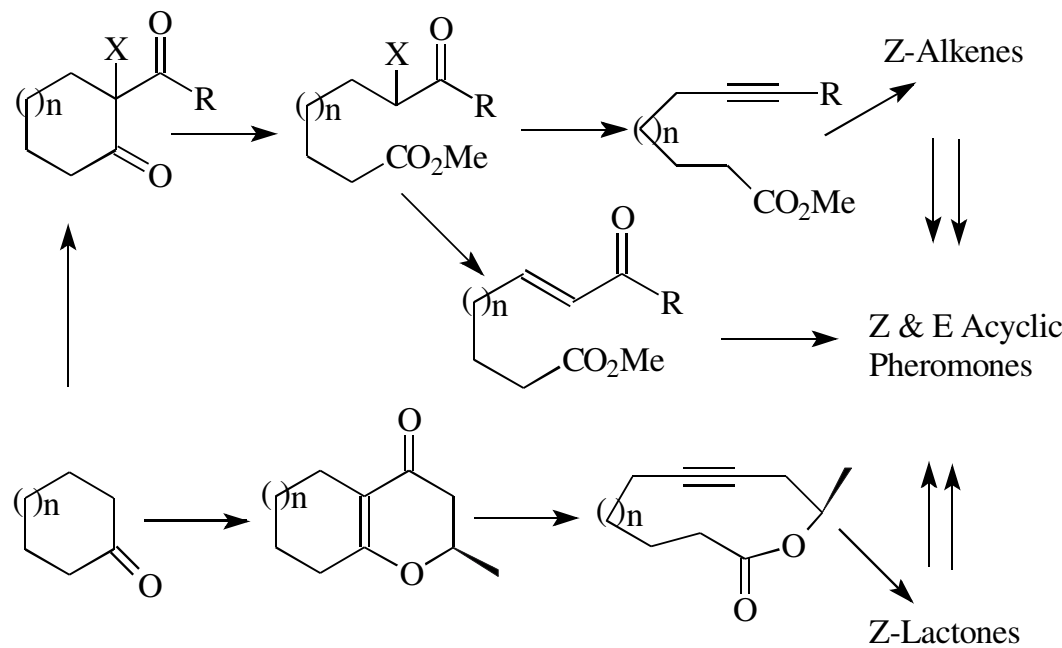

$\mathrm{n}$ : Variable; $\quad \mathrm{X}=\mathrm{Cl}, \mathrm{Br}, \phi \mathrm{S}, \phi \mathrm{Se}$

Scheme 1. General Strategies for the Synthesis of Acyclic Pheromones from Cycloalkanones.

members of the title compounds and their geometric or positional isomers, as illustrated in Scheme 2.

\section{Results and Discussion}

Methanolysis of the Z-lactones ${ }^{6}$ 2a-d gave the corresponding hydroxy-esters $3 \mathrm{a}-\mathrm{d}$, which on mesylation ${ }^{8}$ furnished the respective mesylates $4 \mathrm{a}-\mathrm{d}$. Lithium aluminum hydride reduction of the latter in ether afforded the Z-alkenols 5a-d, which were acetylated to the corresponding acetates $6 \mathrm{a}-\mathrm{d}$. Of these, $(\mathrm{Z})$-5-decenyl acetate (6a) is the pheromone component of the turnip moth (Agrotis segetum $)^{3}$, while $(\mathrm{Z})$-8-dodecenyl acetate $(6 \mathrm{c})$ constitutes the sex pheromone of the oriental fruit moth (Grapholita molesta $)^{3}$. These two compounds have been synthesized earli$\mathrm{er}^{3}$.

Both the catalytic isomerization $\left(\mathrm{NaNO}_{2}, \mathrm{HNO}_{3}\right)^{9}$ and the chemical inversion (TFA, NBS; NaI, DMF, $\Delta)^{10}$ of the $\mathrm{Z}$ alkenyl acetates $\mathbf{6 a - d}$ worked well with these acyclic compounds, leading to the corresponding E acetates 8a-d in good (90-95\%) yield. Nevertheless, the catalytic technique $^{9}\left(2 \mathrm{M} \mathrm{NaNO}_{2}, 2 \mathrm{M} \mathrm{HNO}_{3}, 70-75^{\circ} \mathrm{C}, 1 \mathrm{~h}\right)$ is far more convenient and economical. Among the $\mathrm{E}$ compounds 8a-d, only (E)-8-dodecenyl acetate (8c) is a minor component of the pheromone of the oriental fruit fly (Grapholita molesta), mentioned earlier ${ }^{3}$.

We would like to stress here that the alternative route to these E alkenyl acetates 8a-d, through the respective transformations of the E lactones (dashed arrows in Scheme 2-A), is rather limited and less attractive, because the $\mathrm{Z}$ to $\mathrm{E}$ isomerization works better in the acyclic compounds as compared with the cyclic ones (lactones), as discussed elsewhere ${ }^{6}$.

Another path was tested with the opening of the acetylenic lactone 1b. However, reduction of the mesiloxy-ester 13b, with $\mathrm{LiAlH}_{4}$ in ether, required a much lower initial reaction temperature $\left(-100^{\circ} \mathrm{C}\right.$ as compared with the usual -15 ${ }^{\circ} \mathrm{C}$ ), but still gave a mixture of the desired alcohol $\mathbf{1 4 b}$ and the unwanted diol $\mathbf{1 6 b}$. The latter could be separated by column chromatography of the corresponding acetates $\mathbf{1 5 b}$ and 17b. Despite this drawback, this path has the advantage of leading to both the $\mathrm{Z}$ and $\mathrm{E}$ isomers directly from the acetylenic precursor. Thus, semi-hydrogenation $\left(\mathrm{H}_{2}\right.$, Lindlar cat.) of 15b furnished (Z)-6-decenyl acetate (6b), while its trans reduction with $\mathrm{LiAlH}_{4}$ in refluxing diglyme ${ }^{11}$ gave 
directly (E)-6-decen-1-ol (7b), which was converted into the respective acetate $\mathbf{8 b}$ (Scheme 2-B).

Apart from furnishing the acetylenic intermediates as well as both the $\mathrm{Z}$ and $\mathrm{E}$ alkenyl alcohols and their esters, the present methodology also opens an access to the saturated pheromones, either by the full hydrogenation of the un- saturated precursores or by the systematic elaboration of the saturated lactones. We chose to prepare decyl acetate (18) and hexadecyl acetate (19), pheromone components of the turnip moth (Agrotis segetum) ${ }^{12}$ and the male butterfly Lycorea ceres ceres ${ }^{13}$, respectively, from the corresponding Z-alkenyl acetates (Scheme 2-C).

A: Via Ethylenic Macrolides

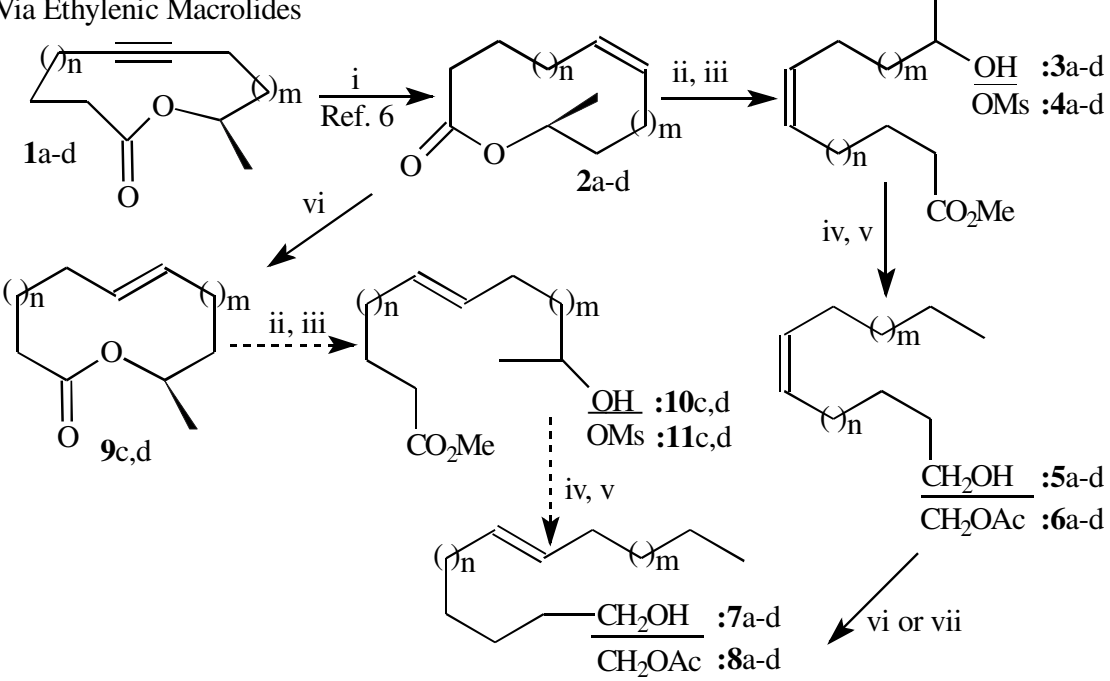

B: From 6-Decyn-9-olide (1b)

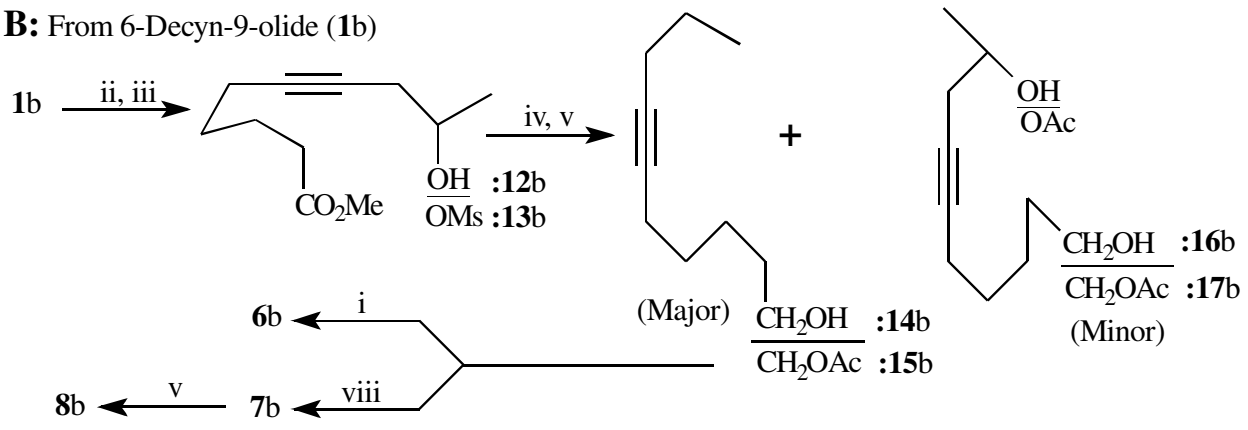

C: Saturated Alkyl Acetates

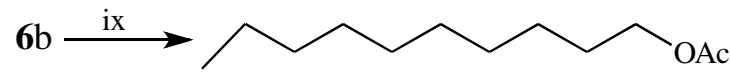

18: Decyl Acetate

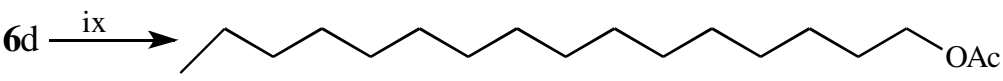

19: Hexadecyl Acetate

$$
\text { a: } m=n=1 ; \quad b: m=o, n=2 ; \quad c: m=0, n=4 ; \quad d: m=0, n=8
$$

Scheme 2. Preparation of $Z$ \& E Pheromones from Acetylenic Lactones. Reagents: i) Lindlar cat., $\mathrm{H}_{2}$; ii) MeOH, MeONa, reflux, 20-24 h; iii) $\mathrm{MsCl}$, $\mathrm{Et}_{3} \mathrm{~N}$, $\mathrm{CH}_{2} \mathrm{Cl}_{2},-15^{\circ} \mathrm{C}, 3 \mathrm{~h}$; iv) $\mathrm{LiAlH}_{4}$, ether, $-15^{\circ} \mathrm{C}$ to reflux, $14-16 \mathrm{~h}$; v) $\mathrm{Ac}_{2} \mathrm{O}$, Py (cat.), $80-90^{\circ} \mathrm{C}, 3 \mathrm{~h}$; vi) $\mathrm{NaNO}_{2}, \mathrm{HNO}_{3}, 70-75^{\circ} \mathrm{C}, 1 \mathrm{~h}$; vii) NBS, TFA, $0^{\circ} \mathrm{C}, 30$ min; NaI, DMF, $90{ }^{\circ} \mathrm{C}, 20-22 \mathrm{~h}$; viii) $\mathrm{LiAlH}_{4}$, diglyme, reflux, $24 \mathrm{~h}$; ix) Pd-C, $\mathrm{H}_{2}$. All reactions gave $90-95 \%$ crude yields (see Experimental). 
The infrared and $90 \mathrm{MHz}{ }^{1} \mathrm{H}$ NMR spectra do not clearly differentiate between the $\mathrm{Z}$ and $\mathrm{E}$ alkenyl acetates (6a-d / 8a-d). Consequently, we resorted to their 200/50 $\mathrm{MHz}{ }^{1} \mathrm{H}$ - and ${ }^{13} \mathrm{C}$ - spectra (PND, DEPT, ${ }^{1} \mathrm{H} \mathrm{x}{ }^{1} \mathrm{H}$ and ${ }^{1} \mathrm{H} \mathrm{x}{ }^{13} \mathrm{C}$ COSY, etc.), where the most evident distinguishing feature, as expected, was the chemical shift of the allylic carbon atoms, which are 4 to $6 \mathrm{ppm}$ upfield in the $\mathrm{Z}$ compounds as compared with the respective atoms in the $\mathrm{E}$ isomers (Tables 1 and 2) ${ }^{14}$. Moreover, close examination of the olefinic carbon atoms in the PND spectra revealed that all the E alkenyl acetates (8a-d) were contaminated with $5-10 \%$ of the corresponding $\mathrm{Z}$ isomer, while only the (Z)-8-dodecenyl acetate (6c) and (Z)-12-hexadecenyl acetate $(\mathbf{6 d})$ contained 5-10\% of the respective E compound, as also present in the precursor Z-lactones ${ }^{6} \mathbf{2 c}, \mathbf{d}$.

\section{Experimental}

Reagent grade chemicals and solvents were used as received from the commercial suppliers, unless noted otherwise. All reactions were monitored routinely by thin layer chromatography (TLC: silica gel, revealed by $\mathrm{I}_{2}$ vapours). Organic extracts were dried over anhydrous $\mathrm{Na}_{2} \mathrm{SO}_{4}$ and evaporated under reduced pressure on a rotary evaporator. IR spectra of all liquid samples were recorded on a Nicolet 5ZDX-FT spectrometer as neat films. Routine ${ }^{1} \mathrm{H}-\mathrm{NMR}$ spectra, reported in the experimental text, were obtained on a Varian EM-390 (90 MHz) instrument as $\mathrm{CCl}_{4}$ solutions, while the high resolution ${ }^{1} \mathrm{H}$ - and ${ }^{13} \mathrm{C}$ - spectra (PND, DEPT, ${ }^{1} \mathrm{Hx}^{1} \mathrm{H}$ and ${ }^{1} \mathrm{Hx}^{13} \mathrm{C}$ COSY, etc.) for the Z- and E-alkenyl acetates (Tables 1 and 2) were recorded in $\mathrm{CDCl}_{3}$ on a Bruker AC-200 (200/50 MHz) spectrometer. Bransonic ultrasonic cleaner (Model 1210 or 2210; $47 \pm 6 \mathrm{KHz}$ ) was used to conduct the semi-hydrogenations. Temperatures in the short path distillations refer to the air bath. Other experimental details are given below.

\section{Methanolysis of Z-Lactones $\mathbf{2 a - d}$. General procedure}

A solution of 2a-d (5 mmol) in $\mathrm{MeOH}(20 \mathrm{ml})$, containing $\mathrm{NaOMe}(10 \mathrm{~mL}, 0.13 \mathrm{mM})$ and protected with $\mathrm{a} \mathrm{CaCl}_{2}$ tube, was refluxed $\left(\mathrm{N}_{2}\right)$ for 20-24 h. After evaporating excess of the solvent, distilled water $(20 \mathrm{ml})$ was added and the mixture extracted with ethyl acetate $(3 \times 30 \mathrm{ml})$. The combined extract was washed with water $(20 \mathrm{ml})$ and brine $(2 \times 20 \mathrm{~mL})$. Drying and evaporation of the solvent furnished the crude hydroxy-esters 3a-d as yellowish liquids $(\sim 4.5 \mathrm{mmol} ; \sim 90 \%)$, practically pure by TLC, which were used in the next step, after the usual spectral identification.

\section{Methyl (Z)-9-Hydroxy-5-decenoate 3a}

IR (v): 3419, 1739, 1245, 1220, 1202, $1128 \mathrm{~cm}^{-1}$. ${ }^{1} \mathrm{H}-\mathrm{NMR}(\delta): 1.16\left(\mathrm{~d}, \mathrm{~J}=6 \mathrm{~Hz}, 3 \mathrm{H}, \mathrm{CH}_{3}\right), 1.2-1.9(\mathrm{~m}, 4 \mathrm{H}$, $\left.2 \mathrm{CH}_{2}\right), 1.9-2,5\left(\mathrm{~m}, 6 \mathrm{H}, 3 \mathrm{CH}_{2}\right), 3,49(\mathrm{~s}, 1 \mathrm{H}, \mathrm{OH}), 3.6-3.9$ (m, $4 \mathrm{H}$, containing a singlet at $3.65, \mathrm{OCH}_{3}$ and $\mathrm{CH}$ ), 5.15-5.65 ( $\mathrm{m}, 2 \mathrm{H}$, olefinic).

\section{Methyl (Z)-9-Hydroxy-6-decenoate $3 b$}

IR (v): 3496, 1740, 1261, 1209, $1120 \mathrm{~cm}^{-1} .{ }^{1} \mathrm{H}-\mathrm{NMR}$

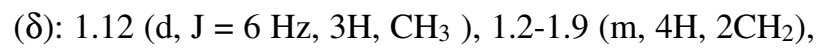
1.9-3.0 $\left(\mathrm{m}, 7 \mathrm{H}, 3 \mathrm{CH}_{2}\right.$ and $\left.\mathrm{OH}\right), 3.5-3.9(\mathrm{~m}, 4 \mathrm{H}$, containing a singlet at $3.62, \mathrm{OCH}_{3}$ and $\left.\mathrm{CH}\right), 5.2-5.7(\mathrm{~m}, 2 \mathrm{H}$, olefinic).

\section{Methyl (Z)-11-Hydroxy-8-dodecenoate 3c}

IR (v): 3425, 1740, 1254, 1201, $1119 \mathrm{~cm}^{-1} .{ }^{1} \mathrm{H}-\mathrm{NMR}$

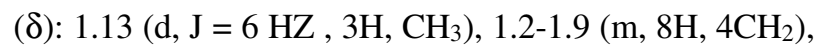
$1.9-2.5\left(\mathrm{~m}, 6 \mathrm{H}, 3 \mathrm{CH}_{2}\right), 3.3(\mathrm{~s}, 1 \mathrm{H}, \mathrm{OH}), 3.6-4.1(\mathrm{~m}, 4 \mathrm{H}$, containing a singlet at $3.62, \mathrm{OCH}_{3}$ and $\left.\mathrm{CH}\right), 5.3-5.7(\mathrm{~m}$, $2 \mathrm{H}$, olefinic).

\section{Methyl (Z)-15-Hydroxy-12-hexadecenoate 3d}

IR (v): 3435, 1740, 1253, 1208, $1120 \mathrm{~cm}^{-1}$. ${ }^{1} \mathrm{H}-\mathrm{NMR}$

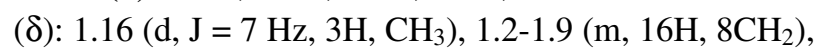
1.9-2.5 (m, 6H, 3CH $), 2.77(\mathrm{~s}, 1 \mathrm{H}, \mathrm{OH}), 3.6-4.0(\mathrm{~m}, 4 \mathrm{H}$, containing a singlet at $3.63, \mathrm{OCH}_{3}$ and $\left.\mathrm{CH}\right), 5.25-5.65(\mathrm{~m}$, $2 \mathrm{H}$, olefinic).

\section{Mesylation of the Hydroxy-Esters 3a-d. General procedu-} re

To a stirred solution of the hydroxy-ester 3a-d (4.5 $\mathrm{mmol})$ and triethylamine $(0,95 \mathrm{~mL}, 690 \mathrm{mg}, 6.83 \mathrm{mmol})$ in $\mathrm{CH}_{2} \mathrm{Cl}_{2}(20 \mathrm{~mL})$, cooled to $-15{ }^{\circ} \mathrm{C}$ and under anhydrous conditions, was added slowly a solution of $\mathrm{H}_{3} \mathrm{CSO}_{2} \mathrm{Cl}$ ( $0.4 \mathrm{~mL}, 592 \mathrm{mg}, 5.17 \mathrm{mmol})$ in $\mathrm{CH}_{2} \mathrm{Cl}_{2}(10 \mathrm{~mL})$. The reaction mixture was stirred for $3 \mathrm{~h}$, when it was diluted with more $\mathrm{CH}_{2} \mathrm{Cl}_{2}(50 \mathrm{~mL})$ and washed with water $(3 \times 20 \mathrm{~mL})$ and brine $(20 \mathrm{~mL})$. Drying and evaporation of solvent gave yellowish, viscous liquids 4a-d (4.0-4.3 mmol; 90-95\%), showing one spot on TLC plates. A rapid passage through a small column of Florisil (3-5 g), in $\mathrm{CH}_{2} \mathrm{Cl}_{2}$ (20-30 mL), afforded purer samples which were characterized by their spectra and subsequently reduced with $\mathrm{LiAlH}_{4}$.

\section{Methyl (Z)-9-Mesyloxy-5-decenoate 4a}

IR (v): 1736, 1354, $1174 \mathrm{~cm}^{-1} .{ }^{1} \mathrm{H}-\mathrm{NMR}(\delta): 1.38(\mathrm{~d}$, $\left.\mathrm{J} \sim 6 \mathrm{~Hz}, 3 \mathrm{H}, \mathrm{CH}_{3}\right), 1.4-1.9\left(\mathrm{~m}, 4 \mathrm{H}, 2 \mathrm{CH}_{2}\right), 1.9-2.5(\mathrm{~m}, 6 \mathrm{H}$, $\left.3 \mathrm{CH}_{2}\right), 3.0\left(\mathrm{~s}, 3 \mathrm{H}, \mathrm{SO}_{3} \mathrm{CH}_{3}\right), 3.62\left(\mathrm{~s}, 3 \mathrm{H}, \mathrm{OCH}_{3}\right), 4,75$ (sextet, $\mathrm{J} \sim 6 \mathrm{~Hz}, 1 \mathrm{H}, \mathrm{CH}), 5.2-5.6(\mathrm{~m}, 2 \mathrm{H}$, olefinic).

\section{Methyl (Z)-9-Mesyloxy-6-decenoate $\mathbf{4 b}$}

IR (v): 1737, 1355, 1207, $1177 \mathrm{~cm}^{-1} .{ }^{1} \mathrm{H}-\mathrm{NMR}(\delta)$ : 1.1-1.9 (m, $7 \mathrm{H}$, containing a doublet at $1.39, \mathrm{~J} \sim 6 \mathrm{~Hz}, \mathrm{CH}_{3}$ and $\left.2 \mathrm{CH}_{2}\right), 1.9-2.7\left(\mathrm{~m}, 6 \mathrm{H}, 3 \mathrm{CH}_{2}\right), 2.97\left(\mathrm{~s}, 3 \mathrm{H}, \mathrm{SO}_{3} \mathrm{CH}_{3}\right)$, $3.63\left(\mathrm{~s}, 3 \mathrm{H}, \mathrm{OCH}_{3}\right), 4.75$ (sextet, $\mathrm{J} \sim 6 \mathrm{~Hz}, 1 \mathrm{H}, \mathrm{CH}$ ), 5.2-5.8 (m, 2H, olefinic). 


\section{Methyl (Z)-11-Mesyloxy-8-dodecenoate $4 c$}

IR (v): 1737, 1357, 1253, $1176 \mathrm{~cm}^{-1} .{ }^{1} \mathrm{H}-\mathrm{NMR}(\delta)$ : 1.1-1.9 (m, 11H, containing a doublet at $1.4, \mathrm{~J} \sim 6 \mathrm{~Hz}, \mathrm{CH}_{3}$ and $\left.4 \mathrm{CH}_{2}\right), 1.9-2.7\left(\mathrm{~m}, 6 \mathrm{H}, 3 \mathrm{CH}_{2}\right), 2.95\left(\mathrm{~s}, 3 \mathrm{H}, \mathrm{SO}_{3} \mathrm{CH}_{3}\right)$, $3.63\left(\mathrm{~s}, 3 \mathrm{H}, \mathrm{OCH}_{3}\right), 4,75$ (sextet, $\left.\mathrm{J} \sim 6 \mathrm{~Hz}, 1 \mathrm{H}, \mathrm{CH}\right), 5.2-5.8$ (m, 2H, olefinic).

\section{Methyl (Z)-15-Mesyloxy-12-hexadecenoate 4d}

IR (v): 1738, 1358, 1252, $1176 \mathrm{~cm}^{-1} .{ }^{1} \mathrm{H}-\mathrm{NMR}(\delta)$ : 1.1-1.9 (m, 19H, containing a doublet at $1.37, \mathrm{~J} \sim 7 \mathrm{~Hz}, \mathrm{CH}_{3}$ and $\left.8 \mathrm{CH}_{2}\right), 1.9-2.7\left(\mathrm{~m}, 6 \mathrm{H}, 3 \mathrm{CH}_{2}\right), 2.97\left(\mathrm{~s}, 3 \mathrm{H}, \mathrm{SO}_{3} \mathrm{CH}_{3}\right)$, $3.6\left(\mathrm{~s}, 3 \mathrm{H}, \mathrm{OCH}_{3}\right), 4.75$ (sextet, $\left.\mathrm{J} \sim 6 \mathrm{~Hz}, 1 \mathrm{H}, \mathrm{CH}\right), 5.2-5.8$ (m, 2H, olefinic).

\section{Reduction of Mesyloxy-esters 4a-d. General procedure}

To a stirred suspension of $\mathrm{LiAlH}_{4}(608-646 \mathrm{mg}, 16-17$ mmol) in ether (40-50 mL), cooled to $-15^{\circ} \mathrm{C}$, was added dropwise a solution of the mesyloxy-ester 4 a-d $(4.0-4.3 \mathrm{mmol})$ in the same solvent $(10 \mathrm{~mL})$. The reaction mixture was stirred until it attained r. t. (2-3 h), when it was gently refluxed for 14-16 h. After cooling, it was carefully decomposed with small pieces of ice and extracted with ethyl acetate $(3 \times 30 \mathrm{~mL})$, the combined extract being successively washed with dil. $\mathrm{HCl}(20 \mathrm{~mL})$, satd. solution of $\mathrm{NaHCO}_{3}(20 \mathrm{~mL})$ and brine $(20 \mathrm{~mL})$. Drying and evaporation furnished yellowish liquids (3.6-3.8 mmol; 90-95\%), which were acetylated after spectral characterization.

\section{(Z)-5-Decenol 5a}

IR (v): 3336, $1056 \mathrm{~cm}^{-1} .{ }^{1} \mathrm{H}-\mathrm{NMR}(\delta): 0.94$ (deformed t, $\left.3 \mathrm{H}, \mathrm{CH}_{3}\right), 1.1-1.8\left(\mathrm{~m}, 8 \mathrm{H}, 4 \mathrm{CH}_{2}\right), 1.8-2.4\left(\mathrm{~m}, 4 \mathrm{H}, 2 \mathrm{CH}_{2}\right)$, $3.56\left(\mathrm{t}, \mathrm{J}=6 \mathrm{~Hz}, 2 \mathrm{H}, \mathrm{CH}_{2} \mathrm{O}\right), 4.5$ (br. s, $\left.1 \mathrm{H}, \mathrm{OH}\right), 5.15-5.60$ (m, $2 \mathrm{H}$, olefinic).

\section{(Z)-6-Decenol $5 \boldsymbol{b}$}

IR (v): 3338, $1054 \mathrm{~cm}^{-1} .{ }^{1} \mathrm{H}-\mathrm{NMR}(\delta): 0.88(\mathrm{t}, \mathrm{J}=7 \mathrm{~Hz}$, $\left.3 \mathrm{H}, \mathrm{CH}_{3}\right), 1.1-1.8\left(\mathrm{~m}, 8 \mathrm{H}, 4 \mathrm{CH}_{2}\right), 1.8-2.3\left(\mathrm{~m}, 4 \mathrm{H}, 2 \mathrm{CH}_{2}\right)$, $3.5\left(\mathrm{t}, \mathrm{J}=6 \mathrm{~Hz}, 2 \mathrm{H}, \mathrm{CH}_{2} \mathrm{O}\right), 3.8$ (br. s, $\left.1 \mathrm{H}, \mathrm{OH}\right), 5.1-5.5$ (m, $2 \mathrm{H}$, olefinic).

\section{(Z)-8-Dodecenol 5c}

IR (v): 3332, $1058 \mathrm{~cm}^{-1} .{ }^{1} \mathrm{H}-\mathrm{NMR}(\delta): 0.90(\mathrm{t}, \mathrm{J}=7 \mathrm{~Hz}$, $\left.3 \mathrm{H}, \mathrm{CH}_{3}\right), 1.1-1.8\left(\mathrm{~m}, 12 \mathrm{H}, 6 \mathrm{CH}_{2}\right), 1.8-2.3\left(\mathrm{~m}, 4 \mathrm{H}, 2 \mathrm{CH}_{2}\right)$, $3.52\left(\mathrm{t}, \mathrm{J}=6 \mathrm{~Hz}, 2 \mathrm{H}, \mathrm{CH}_{2} \mathrm{O}\right), 4.0$ (br. s, $\left.1 \mathrm{H}, \mathrm{OH}\right), 5.15-5.55$ (m, $2 \mathrm{H}$, olefinic).

\section{(Z)-12-Hexadecenol 5d}

IR (v): 3334, $1056 \mathrm{~cm}^{-1} .{ }^{1} \mathrm{H}-\mathrm{NMR}(\delta): 0.92$ (t, J 7 Hz, $\left.3 \mathrm{H}, \mathrm{CH}_{3}\right), 1.1-1.8\left(\mathrm{~m}, 20 \mathrm{H}, 10 \mathrm{CH}_{2}\right), 1.8-2.3(\mathrm{~m}, 4 \mathrm{H}$, $2 \mathrm{CH}_{2}$ ), 3.52 (t, J = 6 Hz, 2H, $\mathrm{CH}_{2}$ ), 3.93 (br. s, OH), 5.1-5.5 (m, $2 \mathrm{H}$, olefinic).
Acetylation of Z-Alkenols 5a-d. General procedure

A solution of 5a-d (3.6-3.8 mmol) in acetic anhydride (3.2-3.4 mL, 3.5-3.7 g, 34-36 mmol), containing 6-7 drops of pyridine and protected with a $\mathrm{CaCl}_{2}$ tube, was heated on a water bath (80-90 $\left.{ }^{\circ} \mathrm{C}\right)$ for $3 \mathrm{~h}$, after which water (15-20 $\mathrm{mL})$ was added and the cooled reaction mixture extracted with hexane $(3 \times 30 \mathrm{~mL})$. The combined extract was washed with dil. $\mathrm{HCl}(10 \mathrm{~mL})$, satd. solution of $\mathrm{NaHCO}_{3}(10$ $\mathrm{mL})$, and brine $(20 \mathrm{~mL})$. Drying and evaporation gave the desired acetates 6a-d, as colorless liquids, possessing pleasant odor. Short path distillation (6a-c: $110-120{ }^{\circ} \mathrm{C} / 5$ Torr; 6d: $110-120{ }^{\circ} \mathrm{C} / 0.5$ Torr) furnished the pure samples in $85-90 \%$ yield.

\section{(Z)-5-Decenyl Acetate ${ }^{3} \mathbf{6} \boldsymbol{a}$}

Colorless liquid (641 mg; $~ 90 \%$ yield). IR (v): 1745, $1234 \mathrm{~cm}^{-1} .{ }^{1} \mathrm{H}-\mathrm{NMR}(\delta): 0,9$ (deformed t, J $\sim 6 \mathrm{~Hz}, 3 \mathrm{H}$, $\left.\mathrm{CH}_{3}\right), 1.1-1.8\left(\mathrm{~m}, 8 \mathrm{H}, 4 \mathrm{CH}_{2}\right), 1.8-2.5(\mathrm{~m}, 7 \mathrm{H}$, containing a singlet at 1.96, $\mathrm{AcO}$ and $\left.2 \mathrm{CH}_{2}\right), 4,0\left(\mathrm{t}, \mathrm{J}=6 \mathrm{~Hz}, 2 \mathrm{H}, \mathrm{CH}_{2} \mathrm{O}\right)$, 5.15-5.55 (m, 2H, olefinic).

\section{(Z)-6-Decenyl Acetate $\mathbf{6 b}$}

Colorless liquid (652mg; 90\%). IR (v): 1743, 1236 $\mathrm{cm}^{-1} .{ }^{1} \mathrm{H}-\mathrm{NMR}(\delta): 0.92\left(\mathrm{t}, \mathrm{J} \sim 6 \mathrm{~Hz}, 3 \mathrm{H}, \mathrm{CH}_{3}\right), 1.1-1.9(\mathrm{~m}$, $\left.\sim 10 \mathrm{H}, 5 \mathrm{CH}_{2}\right), 1.9-2.4(\mathrm{~m}, \sim 5 \mathrm{H}$, containing a singlet at 2.0 , $\mathrm{AcO}$ and $\left.\mathrm{CH}_{2}\right), 4,0\left(\mathrm{t}, \mathrm{J}=6 \mathrm{~Hz}, 2 \mathrm{H}, \mathrm{CH}_{2} \mathrm{O}\right), 5.1-5.6(\mathrm{~m}, 2 \mathrm{H}$, olefinic).

\section{(Z)-8-Dodecenyl Acetate Ac $^{3}$}

Colorless liquid (691mg; 85\%). IR (v): 1743, 1240 $\mathrm{cm}^{-1} .{ }^{1} \mathrm{H}-\mathrm{NMR}(\delta): 1.0\left(\mathrm{t}, \mathrm{J}=6 \mathrm{~Hz}, 3 \mathrm{H}, \mathrm{CH}_{3}\right), 1.2-1.8(\mathrm{~m}$, $\left.\sim 12 \mathrm{H}, 6 \mathrm{CH}_{2}\right), 1.8-2.5(\mathrm{~m}, \sim 7 \mathrm{H}$, containing a singlet at 2.05, $\mathrm{AcO}$ and $\left.2 \mathrm{CH}_{2}\right), 4,05\left(\mathrm{t}, \mathrm{J}=6 \mathrm{~Hz}, 2 \mathrm{H}, \mathrm{CH}_{2} \mathrm{O}\right)$, 5.2-5.6 (m, $2 \mathrm{H}$, olefinic).

\section{(Z)-12-Hexadecenyl Acetate $6 \mathbf{d}$}

Colorless liquid (873 mg; 86\%). IR (v): 1743, 1237 $\mathrm{cm}^{-1} .{ }^{1} \mathrm{H}-\mathrm{NMR}(\delta): 0.89\left(\mathrm{t}, \mathrm{J} \sim 7 \mathrm{~Hz}, 3 \mathrm{H}, \mathrm{CH}_{3}\right), 1.1-1.8(\mathrm{~m}$, $\left.20 \mathrm{H}, 10 \mathrm{CH}_{2}\right), 1.8-2.6(\mathrm{~m}, 7 \mathrm{H}$, having a singlet at $1.95, \mathrm{AcO}$ and $\left.2 \mathrm{CH}_{2}\right), 4,0\left(\mathrm{t}, \mathrm{J} \sim 7 \mathrm{~Hz}, 2 \mathrm{H}, \mathrm{CH}_{2} \mathrm{O}\right), 5.15-5.60(\mathrm{~m}, 2 \mathrm{H}$, olefinic).

200/50 MHz spectra of these acetates are shown in Table 1 .

Catalytic Isomerization of Z Acetates 6 a-d into E Isomers 8a-d. General procedure

Sodium nitrite solution $(2 \mathrm{M}, 0.1 \mathrm{~mL})$ and $2 \mathrm{M} \mathrm{HNO}_{3}$ $(0.2 \mathrm{~mL})$ were added to the $\mathrm{Z}$ acetate $\mathbf{6 a - d}(1 \mathrm{mmol})$, kept under $\mathrm{N}_{2}$ atmosphere. The resulting yellowish mixture was stirred vigorously and heated to $70-75^{\circ} \mathrm{C}$ for $1 \mathrm{~h}$. After cooling and dilution with water $(10 \mathrm{~mL})$, it was extracted with hexane $(3 \times 20 \mathrm{~mL})$, the combined extract being washed 
Table 1. ${ }^{13} \mathrm{C}$ - and ${ }^{1} \mathrm{H}$ - spectra ${ }^{\#}$ of Z-Alkenyl Acetates 6a-d.

\begin{tabular}{|c|c|c|c|c|c|c|c|c|}
\hline \multirow{2}{*}{$\begin{array}{l}\text { Compound: } \\
\text { C- } \\
\end{array}$} & \multicolumn{2}{|c|}{$6 a$} & \multicolumn{2}{|c|}{$6 \mathbf{b}$} & \multicolumn{2}{|c|}{$6 c$} & \multicolumn{2}{|c|}{ 6d } \\
\hline & $\delta \mathrm{C}$ & $\delta \mathrm{H}$ & $\delta \mathrm{C}$ & $\delta \mathrm{H}$ & $\delta \mathrm{C}$ & $\delta \mathrm{H}$ & $\delta \mathrm{C}$ & $\delta \mathrm{H}$ \\
\hline ACO: & 170.93 & - & 170.88 & - & $\sim 171.0$ & - & 171.06 & - \\
\hline $\mathrm{CH}_{3}$ & 20.79 & $1.96 \mathrm{~s}$ & 20.85 & $1.97 \mathrm{~s}$ & 20.72 & $2.05 \mathrm{~s}$ & 20.84 & $1.98 \mathrm{~s}$ \\
\hline 1 & 64.24 & $3.98 \mathrm{t}$ & 64.43 & $3.97 \mathrm{t}$ & 64.39 & $4.05 \mathrm{t}$ & 64.53 & $4.00 \mathrm{t}$ \\
\hline 2 & 28.03 & $1.5-1.7$ & 28.39 & $1.5-1.7$ & 28.44 & $1.5-1.7$ & 28.51 & $1.56 \mathrm{~m}$ \\
\hline 3 & 25.85 & $1.2-1.4$ & 25.42 & $1.2-1.4$ & 25.73 & $1.2-1.5$ & 25.82 & $1.1-1.4$ \\
\hline 4 & $26.55^{*}$ & $1.99 \mathrm{~m}$ & 29.21 & $1.2-1.4$ & 29.12 & $1.2-1.5$ & $29.19^{\mathrm{i}}$ & $1.1-1.4$ \\
\hline 5 & $128.85^{\mathrm{i}}$ & $5.26 \mathrm{~m}$ & $26.90 *$ & $1.8-2.0$ & 28.99 & $1.2-1.5$ & $29.66^{\mathrm{i}}$ & $1.1-1.4$ \\
\hline 6 & $130.34^{\mathrm{i}}$ & $5.28 \mathrm{~m}$ & 129.83 & $5.1-5.4$ & 28.99 & $1.2-1.5$ & $29.46^{\mathrm{i}}$ & $1.1-1.4$ \\
\hline 7 & $26.76^{*}$ & $1.93 \mathrm{~m}$ & 129.40 & $5.1-5.4$ & $26.99 *$ & $1.9-2.1$ & $29.46^{\mathrm{i}}$ & $1.1-1.4$ \\
\hline 8 & 31.75 & $1.1-1.3$ & $29.21 *$ & $1.8-2.0$ & 129.72 & $5.2-5.5$ & $29.46^{\mathrm{i}}$ & $1.1-1.4$ \\
\hline 9 & $22-18$ & $1.1-1.3$ & 22.75 & $1.2-1.4$ & 129.52 & $5.2-5.5$ & $29.66^{\mathrm{i}}$ & $1.1-1.4$ \\
\hline 10 & 13.80 & $0.82 \mathrm{t}$ & 13.66 & $0.82 \mathrm{t}$ & $29.49 *$ & $1.9-2.1$ & $29.19^{\mathrm{i}}$ & $1.1-1.4$ \\
\hline 11 & - & - & - & - & 22.73 & $1.2-1.5$ & $27.12 *$ & $1.9-2.0$ \\
\hline 12 & - & - & - & - & 13.61 & $0.90 \mathrm{t}$ & 129.96 & $5.3 \mathrm{t}$ \\
\hline 13 & - & - & - & - & - & - & 129.48 & $5.3 \mathrm{t}$ \\
\hline 14 & - & - & - & - & - & - & $29.19 *$ & $1.9-2.0$ \\
\hline 15 & - & - & - & - & - & - & 22.79 & $1.1-1.4$ \\
\hline 16 & - & - & - & - & - & - & 13.69 & $0.85 \mathrm{t}$ \\
\hline
\end{tabular}

\#: Based on PND, DEPT, ${ }^{1} \mathrm{H} \mathrm{x}{ }^{1} \mathrm{H}$ and ${ }^{1} \mathrm{H} \mathrm{x}{ }^{13} \mathrm{C}$ COSY Spectra at $200 / 50 \mathrm{MHz}$ in $\mathrm{CDCl}_{3}$.

*: Allylic C; i: Interchangeable assignment; s, $\mathrm{m}, \mathrm{t}$ : Usual multiplicity of peaks.

with satd. soln. of $\mathrm{NaHCO}_{3}(10 \mathrm{~mL})$ and brine $(10 \mathrm{~mL})$. Drying and evaporation gave yellowish liquids in $95-100 \%$ yield. Short path distillation (8a-c: $110-120{ }^{\circ} \mathrm{C} / 5$ Torr; $8 d$ : $110-120^{\circ} \mathrm{C} / 0.5$ Torr) afforded colorless liquids (90-95\%).

\section{(E)-5-Decenyl Acetate $8 \boldsymbol{a}$}

IR (v): $1744,1240,970 \mathrm{~cm}^{-1} .{ }^{1} \mathrm{H}-\mathrm{NMR}(\delta): 0.90$ (deformed $\left.\mathrm{t}, \mathrm{J} \sim 6 \mathrm{~Hz}, 3 \mathrm{H}, \mathrm{CH}_{3}\right), 1.1-1.9\left(\mathrm{~m}, 8 \mathrm{H}, 4 \mathrm{CH}_{2}\right), 1.9-2.3$ $\left(\mathrm{m}, 7 \mathrm{H}\right.$, having a singlet at $2.03, \mathrm{AcO}$ and $\left.2 \mathrm{CH}_{2}\right), 4.0(\mathrm{t}$, $\left.\mathrm{J}=6 \mathrm{~Hz}, 2 \mathrm{H}, \mathrm{CH}_{2} \mathrm{O}\right), 5.15-5.65$ (m, 2H, olefinic).

\section{(E)-6-Decenyl Acetate $8 \boldsymbol{b}$}

IR (v): 1743, 1238, $969 \mathrm{~cm}^{-1} .{ }^{1} \mathrm{H}-\mathrm{NMR}(\delta): 0.86(\mathrm{t}$, $\left.\mathrm{J} \sim 7 \mathrm{~Hz}, 3 \mathrm{H}, \mathrm{CH}_{3}\right), 1.1-1.8\left(\mathrm{~m}, 8 \mathrm{H}, 2 \mathrm{CH}_{2}\right), 1.8-2.3(\mathrm{~m}, 7 \mathrm{H}$, showing a singlet at $1.95, \mathrm{AcO}$ and $\left.2 \mathrm{CH}_{2}\right), 3.98(\mathrm{t}, \mathrm{J} \sim 6 \mathrm{~Hz}$, $\left.2 \mathrm{H}, \mathrm{CH}_{2} \mathrm{O}\right)$, 5.1-5.6 (m, 2H, olefinic).

\section{(E)-8-Dodecenyl Acetate ${ }^{3} 8 \boldsymbol{c}$}

IR (v): 1743, 1240, $969 \mathrm{~cm}^{-1} .{ }^{1} \mathrm{H}-\mathrm{NMR}(\delta): 0.94(\mathrm{t}, \mathrm{J} \sim 7$ $\left.\mathrm{Hz}, 3 \mathrm{H}, \mathrm{CH}_{3}\right), 1.20-1.85\left(\mathrm{~m}, 12 \mathrm{H}, 6 \mathrm{CH}_{2}\right), 1.85-2.5(\mathrm{~m}, 7 \mathrm{H}$, having a singlet at 2.02 , AcO and $\left.2 \mathrm{CH}_{2}\right), 4.04(\mathrm{t}, \mathrm{J}=6 \mathrm{~Hz}$, $\left.\left.2 \mathrm{H}, \mathrm{CH}_{2}\right)\right)$, 5.2-5.6 (m, $2 \mathrm{H}$, olefinic).

(E)-12-Hexadecenyl Acetate 8d
IR (v): $1743,1238,967 \mathrm{~cm}^{-1} .{ }^{1} \mathrm{H}-\mathrm{NMR}(\delta): 0.88$ (deformed t, $\left.3 \mathrm{H}, \mathrm{CH}_{3}\right), 1.1-1.8\left(\mathrm{~m}, 20 \mathrm{H}, 10 \mathrm{CH}_{2}\right), 1.8-2.6(\mathrm{~m}, 7 \mathrm{H}$, having a singlet at $1.97, \mathrm{AcO}$ and $\left.2 \mathrm{CH}_{2}\right), 4.0(\mathrm{t}, \mathrm{J}=6 \mathrm{~Hz}$, $2 \mathrm{H}, \mathrm{CH}_{2} \mathrm{O}$ ), 5.15-5.65 ( $\mathrm{m}, 2 \mathrm{H}$, olefinic).

Higher field spectra of these acetates are shown in Table 2 .

\section{Chemical Inversion of Z Acetates (6a-d) to E Isomers} $(8 a-d)$. General procedure

Compound 6a-d ( $0.5 \mathrm{mmol})$ was added slowly to a cold $\left(0{ }^{\circ} \mathrm{C}\right)$ and stirred solution of NBS $(107 \mathrm{mg}, 0.6 \mathrm{mmol})$ in TFA $(0.75 \mathrm{~mL}, 1.1 \mathrm{~g}, 9.74 \mathrm{mmol})$, protected with a drying tube. After stirring for $30 \mathrm{~min}$, the reaction mixture was diluted with water $(10 \mathrm{~mL})$ and extracted with hexane $(3 \times 10$ $\mathrm{mL})$, the extract being washed with water $(5 \times 10 \mathrm{~mL})$ and brine $(10 \mathrm{~mL})$. Usual work-up furnished the crude adducts, in almost quantitative yield, showing characteristic absorptions around 1785 and $1740 \mathrm{~cm}^{-1}$.

The crude product dissolved in DMF $(2 \mathrm{~mL})$ and containing NaI (338 mg, $2.25 \mathrm{mmol})$ was heated at $90{ }^{\circ} \mathrm{C}$ for 20-22 h. After cooling, a dil. solution of $\mathrm{NaHSO}_{3}(10 \mathrm{~mL})$ was added and the whole extracted with hexane ( 3 x $10 \mathrm{~mL}$ ). Usual work-up gave brown liquids, which on short path distillation furnished the E acetates 8a-d, in 
Table 2. ${ }^{13} \mathrm{C}$ - and ${ }^{1} \mathrm{H}$ - spectra ${ }^{\#}$ of E-Alkenyl Acetates 8a-d.

\begin{tabular}{lcccccccc}
\hline Compound: & \multicolumn{3}{c}{$\mathbf{8 a}$} & \multicolumn{3}{c}{$\mathbf{8 b}$} & \multicolumn{3}{c}{$\mathbf{8 c}$} & \multicolumn{3}{c}{$\mathbf{8 d}$} \\
\hline $\mathrm{C}-$ & $\delta \mathrm{C}$ & $\delta \mathrm{H}$ & $\delta \mathrm{C}$ & $\delta \mathrm{H}$ & $\delta \mathrm{C}$ & $\delta \mathrm{H}$ & $\delta \mathrm{C}$ & $\delta \mathrm{H}$ \\
\hline $\mathrm{AcO}:$ & 170.69 & - & $\sim 171.0$ & & 171.06 & - & 170.51 & - \\
$\mathrm{CH}_{3}$ & 20.57 & $2.02 \mathrm{~s}$ & 20.84 & $2.04 \mathrm{~s}$ & 20.93 & $2.05 \mathrm{~s}$ & 20.68 & $2.01 \mathrm{~s}$ \\
1 & 64.10 & $4.03 \mathrm{t}$ & 64.47 & $4.05 \mathrm{t}$ & 64.57 & $4.05 \mathrm{t}$ & 64.35 & $4.01 \mathrm{t}$ \\
2 & 27.79 & $1.5-1.7$ & 28.36 & $1.5-1.7$ & 28.52 & $1.5-1.7$ & 28.46 & $1.53 \mathrm{~m}$ \\
3 & 25.60 & $1.4 \mathrm{~m}$ & 25.27 & $1.1-1.4$ & 25.81 & $1.1-1.5$ & 25.76 & $1.2-1.5$ \\
4 & $31.99^{*}$ & $1.9-2.1$ & 29.09 & $1.1-1.4$ & 29.46 & $1.1-1.5$ & $29.45^{\mathrm{i}}$ & $1.2-1.5$ \\
5 & $130.72^{\mathrm{i}}$ & $5.2-5.5$ & $32.30^{*}$ & $1.8-2.1$ & 29.05 & $1.1-1.5$ & $29.00^{\mathrm{i}}$ & $1.2-1.5$ \\
6 & $129.21^{\mathrm{i}}$ & $5.2-5.5$ & 130.39 & $5.3-5.5$ & 28.93 & $1.1-1.5$ & $29.45^{\mathrm{i}}$ & $1.2-1.5$ \\
7 & $31.85^{*}$ & $1.9-2.1$ & 129.97 & $5.3-5.5$ & $32.49^{*}$ & $1.8-2.1$ & $29.00^{\mathrm{i}}$ & $1.2-1.5$ \\
8 & 31.49 & $1.2-1.4$ & $34.58^{*}$ & $1.8-2.1$ & 130.36 & $5.2-5.5$ & $29.45^{\mathrm{i}}$ & $1.2-1.5$ \\
9 & 21.91 & $1.2-1.4$ & 22.60 & $1.1-1.4$ & 130.16 & $5.2-5.5$ & $29.00^{\mathrm{i}}$ & $1.2-1.5$ \\
10 & 13.63 & $0.87 \mathrm{t}$ & 13.52 & $0.88 \mathrm{t}$ & $34.64^{*}$ & $1.8-2.1$ & $29.45^{\mathrm{i}}$ & $1.2-1.5$ \\
11 & - & - & - & - & 22.63 & $1.1-1.5$ & $32.45^{*}$ & $1.8-2.1$ \\
12 & - & - & - & - & 13.58 & $0.88 \mathrm{t}$ & 129.85 & $5.2-5.4$ \\
13 & - & - & - & - & - & - & 130.32 & $5.2-5.4$ \\
14 & - & - & - & - & - & - & $34.55^{*}$ & $1.8-2.1$ \\
15 & - & - & - & - & - & - & 22.57 & $1.2-1.5$ \\
16 & - & - & - & - & - & - & 13.75 & $0.85 \mathrm{t}$ \\
\hline
\end{tabular}

\#: Based on PND, DEPT, ${ }^{1} \mathrm{H} \mathrm{x}{ }^{1} \mathrm{H}$ and ${ }^{1} \mathrm{H} \mathrm{x}{ }^{13} \mathrm{C}$ COSY Spectra at $200 / 50 \mathrm{MHz}$ in $\mathrm{CDCl}_{3}$.

*: Allylic C; i: Interchangeable assignment; s, m, t : Usual multiplicity of signals.

90-95\% yield, as colorless liquids, identical (IR and ${ }^{1} \mathrm{H}-\mathrm{NMR}$ ) with the respective products obtained earlier by the catalytic procedure.

\section{Methanolysis of the Acetylenic Lactone $\mathbf{1 b}$}

A solution of $\mathbf{1 b}(830 \mathrm{mg}, 5 \mathrm{mmol})$ in $\mathrm{MeOH}(20 \mathrm{~mL})$, containing NaOMe $(0.13 \mathrm{mM}, 10 \mathrm{~mL})$, was refluxed for 2-3 h, when it was worked-up as described earlier for the methanolysis of $\mathrm{Z}$ lactones, obtaining the hydroxy-ester 12b, a yellowish liquid, in $90 \%$ yield $(891 \mathrm{mg})$. IR (v): 3433, 1739, 1283, 1264, 1216, $1177 \mathrm{~cm}^{-1} .{ }^{1} \mathrm{H}-\mathrm{NMR}(\delta): 1.2$ (d, J $\left.=6 \mathrm{~Hz}, 3 \mathrm{H}, \mathrm{CH}_{3}\right), 1.3-2.0\left(\mathrm{~m}, 4 \mathrm{H}, 2 \mathrm{CH}_{2}\right), 2.0-2.6$ (m, $\left.6 \mathrm{H}, 3 \mathrm{CH}_{2}\right), 3.2-4.2\left(\mathrm{~m}, 5 \mathrm{H}\right.$, having a singlet at $3.64, \mathrm{OCH}_{3}$, $\mathrm{CH}, \mathrm{OH})$.

The crude product $(\sim 890 \mathrm{mg})$ was mesylated with $\mathrm{MsCl}$ $(0.4 \mathrm{~mL}, 592 \mathrm{mg}, 5.17 \mathrm{mmol})$ and $\mathrm{Et}_{3} \mathrm{~N}(0.95 \mathrm{~mL})$ in $\mathrm{CH}_{2} \mathrm{Cl}_{2}$ (30 mL), as described earlier, to obtain the mesyloxy-ester $13 \mathrm{~b}$ as a yellowish liquid $(1.24 \mathrm{~g} ; 100 \%)$. After a quick passage through a small column of Florisil ${ }^{\circledR}$ (3-5 g) in $\mathrm{CH}_{2} \mathrm{Cl}_{2}(20-30 \mathrm{~mL})$, it was characterized by its spectra. IR (v): 1736, 1354, 1212, $1176 \mathrm{~cm}^{-1} .{ }^{1} \mathrm{H}-\mathrm{NMR}(\delta): 1.3-2.0$ $\left(\mathrm{m}, 7 \mathrm{H}\right.$, showing a doublet at $1.52, \mathrm{~J}=6 \mathrm{~Hz}, \mathrm{CH}_{3}$ and $\left.2 \mathrm{CH}_{2}\right), 2.0-2.5\left(\mathrm{~m}, 4 \mathrm{H}, 2 \mathrm{CH}_{2}\right), 2.5-2.8\left(\mathrm{~m}, 2 \mathrm{H}, \mathrm{CH}_{2}\right), 3.08$ (s, $3 \mathrm{H}, \mathrm{SO}_{3} \mathrm{CH}_{3}$ ), $3.68\left(\mathrm{~s}, \mathrm{OCH}_{3}\right), 4.8($ sextet, $\mathrm{J}=6 \mathrm{~Hz}, 1 \mathrm{H}$, $\mathrm{CH})$.

\section{Reduction of the Mesyloxy-Ester $13 b$}

A solution of ester $\mathbf{1 3 b}(1.2 \mathrm{~g} ; 4.5 \mathrm{mmol})$ in anhydrous ether $(40 \mathrm{~mL})$ was added slowly to a stirred suspension of $\mathrm{LiAlH}_{4}(684 \mathrm{mg}, 18 \mathrm{mmol})$ in ether $(10 \mathrm{~mL})$, kept at $-100{ }^{\circ} \mathrm{C}$ (liquid $\mathrm{N}_{2}$ and EtOAc). After allowing the reaction mixture to attain r. t. (2-3 h), it was gently refluxed for 14-16 h. Usual work-up, as described in the earlier cases, furnished a yellowish liquid $(770 \mathrm{mg})$, showing two principal spots on TLC plate. Spectral analysis revealed these as the desired alcohol 14b and unwanted diol 16b. IR (v): 3349, 1161, 1073, $1052 \mathrm{~cm}^{-1} .{ }^{1} \mathrm{H}-\mathrm{NMR}(\delta): 0,97(\mathrm{t}, \mathrm{J} \sim 7$ $\left.\mathrm{Hz}, \underline{\mathrm{H}}_{3} \mathrm{C}-\mathrm{CH}_{2}\right), 1.2$ (d, J $\left.6 \mathrm{~Hz}, \underline{\mathrm{H}}_{3} \mathrm{C}-\mathrm{CH}-\mathrm{O}\right)$ 1.2-2.0 (m, methylenes), 2.0-3.0 (m, methylenes), $3.53\left(\sim \mathrm{t}, \mathrm{CH}_{2} \mathrm{O}\right.$ ), $3.7-4.0(\mathrm{~m}$, having a singlet at $3.8, \mathrm{OH}$ and $\mathrm{CH})$.

The above mixture was acetylated with $\mathrm{Ac}_{2} \mathrm{O}(4.3 \mathrm{~mL})$ and pyridine (8 drops), under the usual conditions, and the resulting product $(1.1 \mathrm{~g})$ chromatographed, under reduced pressure, ${ }^{15}$ over silica gel $60 \mathrm{G}(15 \mathrm{~g})$, eluted with hexane-EtOAc (98:2), obtaining the acetylenic monoacetate 15b (690 mg; 70\%) and diacetate 17b (340 mg; 27\%). 


\section{6-Decynyl Acetate $\mathbf{1 5 b}$ (Colorless liquid)}

IR (v): 1742,1238 cm ${ }^{-1} .{ }^{1} \mathrm{H}-\mathrm{NMR}(\delta): 0.98(\mathrm{t}, \mathrm{J}=6 \mathrm{~Hz}$, $\left.3 \mathrm{H}, \mathrm{CH}_{3}\right), 1.2-1.9\left(\mathrm{~m}, 8 \mathrm{H}, 4 \mathrm{CH}_{2}\right), 1.9-2.5(\mathrm{~m}, 7 \mathrm{H}$, containing a singlet at 2.0, $\mathrm{AcO}$ and $\left.2 \mathrm{CH}_{2}\right), 4.03\left(\mathrm{t}, \mathrm{J}=6 \mathrm{~Hz}, 2 \mathrm{H}, \mathrm{CH}_{2} \mathrm{O}\right)$.

\section{1,9-Diacetoxy-6-decyn $\mathbf{1 7 b}$ (Colorless liquid)}

IR (v): 1740, $1242 \mathrm{~cm}^{-1} .{ }^{1} \mathrm{H}-\mathrm{NMR}(\delta): 1.32(\mathrm{t}, \mathrm{J}=6 \mathrm{~Hz}$, $\left.3 \mathrm{H}, \mathrm{CH}_{3}\right), 1.4-1.9\left(\mathrm{~m}, 6 \mathrm{H}, 3 \mathrm{CH}_{2}\right), 1.9-2.7$ (m, 10H, showing a singlet at 2.03, 2AcO and $\left.2 \mathrm{CH}_{2}\right), 4.05(\mathrm{t}, \mathrm{J}=6 \mathrm{~Hz}$, $2 \mathrm{H}, \mathrm{CH}_{2} \mathrm{O}$ ), 4.9 (sextet, $\mathrm{J}=6 \mathrm{~Hz}, 1 \mathrm{H}, \mathrm{CH}$ ).

Semi-hydrogenation ${ }^{6}$ of 6-Decynyl Acetate (15b)

Compound 15b (196 mg, $1 \mathrm{mmol}$ ), Lindlar catalyst (60 $\mathrm{mg})$ and hexane $(10 \mathrm{~mL})$ were put in a pear-shaped flask, which was connected to a balloon filled with $\mathrm{H}_{2}(\sim 500 \mathrm{~mL})$. After the usual purging with $\mathrm{H}_{2}$ (3 times) the reaction flask was placed in the center of an ultrasound bath and irradiated for $4 \mathrm{~h}$, when there was no more starting alkyne $\mathbf{1 5 b}$, as detected by GC analysis (FFAP column, $200^{\circ} \mathrm{C}$ ). Filtration of the catalyst and evaporation of the solvent gave a colorless liquid, which on short path distillation $\left(110-120^{\circ} \mathrm{C} / 5\right.$ Torr) furnished an analytical sample (194 mg; $98 \%$ ), identical in all respects (TLC, IR, ${ }^{1} \mathrm{H}$-NMR) with (Z)-6-decenyl acetate $(\mathbf{6 b})$, prepared earlier by an alternative route.

\section{Reduction of 6-Decynyl Acetate (15b) to (E)-6-Decenyl Alcohol (7b)}

A solution of compound 15b (196 mg, $1 \mathrm{mmol})$ in diglyme $(5 \mathrm{~mL})$ was added dropwise to a magnetically stirred suspension of $\mathrm{LiAlH}_{4}(152 \mathrm{mg}, 4 \mathrm{mmol})$ in diglyme $(10 \mathrm{~mL})$, cooled to $0{ }^{\circ} \mathrm{C}$. After attaining r. t., the reaction mixture was refluxed gently for $24 \mathrm{~h}$, when it was cooled and decomposed carefully with small pieces of ice. Extraction with hexane $(3 \times 20 \mathrm{~mL}$ ) and usual work-up afforded the (E)-6-decenol (7b), which was acetylated to the corresponding acetate under the usual conditions descrided earlier. Short path distillation (110-120 ${ }^{\circ} \mathrm{C} / 5$ Torr) gave the pure compound $\mathbf{8 b}$ (190 mg; 95\%), identical (TLC, IR, ${ }^{1} \mathrm{H}-\mathrm{NMR}$ ) with the product obtained earlier by the isomerization of $\mathrm{Z}$ isomer $\mathbf{6 b}$.

Decyl Acetate ${ }^{12}$ (18)

A solution of (Z)-6-decenyl acetate (6b: $198 \mathrm{mg}, 1$ $\mathrm{mmol})$ in hexane $(10 \mathrm{~mL})$, containing $10 \% \mathrm{Pd}-\mathrm{C}(20-30$ $\mathrm{mg}$ ), was subjected to hydrogenation (2 atm) in a Parr apparatus for $2-3 \mathrm{~h}$, when there was no more of the starting material. Usual work-up furnished the product in a quantitative yield, while short path distillation (110-120 ${ }^{\circ} \mathrm{C} / 5$ Torr) afforded an analytical sample. IR (v): 1744, $1238 \mathrm{~cm}^{-1}$. ${ }^{1} \mathrm{H}-\mathrm{NMR}(\delta): 0.92$ (deformed t, J $6 \mathrm{~Hz}, 3 \mathrm{H}$, $\left.\mathrm{CH}_{3}\right), 1.1-1.8\left(\mathrm{~m}, 16 \mathrm{H}, 8 \mathrm{CH}_{2}\right), 1.97$ (s, 3H, AcO), $4.0(\mathrm{t}$, $\left.\mathrm{J}=6 \mathrm{~Hz}, 2 \mathrm{H}, \mathrm{CH}_{2} \mathrm{O}\right)$.
Hexadecyl Acetate ${ }^{13}$ (19)

Was obtained from (Z)-12-hexadecenyl acetate (6d), exactly in the manner described above. IR (v): 1744, 1237 $\mathrm{cm}^{-1} .{ }^{1} \mathrm{H}-\mathrm{NMR}(\delta): 0.89$ (deformed t, $\left.3 \mathrm{H}, \mathrm{CH}_{3}\right), 1.28(\sim \mathrm{s}$, $\left.28 \mathrm{H}, 14 \mathrm{CH}_{2}\right), 1.97$ (s, 3H, OAC), $4.0\left(\mathrm{t}, \mathrm{J} \sim 6 \mathrm{~Hz}, 2 \mathrm{H}, \mathrm{CH}_{2} \mathrm{O}\right)$.

\section{Acknowledgments}

We thank the Brazilian National Research Council (CNPq), the University of Brasília (UnB) and the "Fundação de Amparo à Pesquisa - DF" (FAP-DF) for partial support of this work. We also thank Professors R. Braz-Filho and M.G. Carvalho (UFRRJ) for the high resolution and 2D spectra of these compounds.

\section{References}

1. a) Rossi, R. Synthesis 1977, 817; b) Henrick, C.A. Tetrahedron 1977, 33, 1845.

2. Mori, K. Tetrahedron 1989, 45, 3223.

3. Mori, K. In The Total Synthesis of Natural Products, Vol. 9; Apsimon, J.; Ed.; Wiley; New York, 1992, and the pertinent references cited therein.

4. a) Mahajan, J.R. In Organic Synthesis in Brazil: An Overview; Comasseto, J.V.; Ferreira, J.T.B.; Ed.; DOT Editoração Eletrônica \& Multimídia; São Carlos - SP, 1994; pp 79-87; b) Mahajan, J.R. ICOS 10, Bangalore-India, 1994; ABSTRACTS: ISP-27, P 81; c) Mahajan, J.R.; Resck, I.S.; 6th BMOS, USP-SP, 1994; ABSTRACTS: p 94; d) Mahajan, J.R.; J. Braz. Chem. Soc. 1996, 7, 297.

5. a) Mahajan, J.R.; Tresvenzol, L.M.F. J. Braz. Chem. Soc. 1993, 4, 179; b) Mahajan, J.R.; Resck, I.S.; Aspesi, G.H. 17th Annual Meeting of SBQ 1994; Abstracts: QO-041.

6. Mahajan, J.R.; Resck, I.S. Synth. Commun. 1996, 26, 3809.

7. a) Mahajan, J.R.; Resck, I.S. J. Chem. Soc., Chem. Commun. 1993, 1748; b) Resck, I.S. PhD Thesis 1995; QUI-IE-UNB.

8. Crossland, R.K.; Servis, K.L. J. Org. Chem. 1970, 35, 3195.

9. Sonnet, P.E. J. Org. Chem. 1974, 39, 3793.

10. Sonnet, P.E. J. Org. Chem. 1980, 45, 154.

11. Schwarz, M.; Graminski, G.F.; Waters, R.M. J. Org. Chem. 1986, 51, 260.

12. Olsson, A.-M.; Jonsson, J.A.; Thelin, B.; Liljefors, T. J. Chem. Ecol. 1983, 9, 375.

13. a) Meinwald, J.; Meinwald, Y.C. J. Am. Chem. Soc. 1966, 88, 1305; b) Meinwald, J.; Meinwald, Y. C.; Wheeler, J.W.; Eisner, T. Science 1966, 151, 583.

14. Mahajan, J.R.; Resck, I.S.; Braz-Filho, R.; Carvalho, M.G. 5th Meeting of NMR Users (V-AUREMN), Angra dos Reis-RJ, 1995; Abstracts: p 38; , pp 123-132. 